\title{
Publicidad persuasiva del sector de la bollería en televisión
}

Recibido: 03 de octubre de 2016

Aceptado: 24 de abril de 2017

Publicado: 27 de noviembre de 2017
Mónica Matellanes Lazo

mmatellanes@uemc.es

Universidad Europea Miguel de Cervantes (España)

Resumen: En los últimos años han ido surgiendo gran diversidad de productos nuevos en el sector de la bollería infantil, pero existen otros que llevan mucho tiempo en nuestra vida diaria. Pese a ser más los que han fracasado que los que se mantienen, resulta crucial analizar cómo ha evolucionado su publicidad a lo largo del tiempo, sabiendo adaptarse a los gustos de diferentes generaciones de consumidores. Entre los clientes y los consumidores ha surgido claramente un movimiento de preocupación y concienciación por los ingredientes que componen los alimentos. Debido al cambio de actitud y a la proliferación de asociaciones de defensa de los derechos del consumidor, se ha llevado a cabo una evolución en este tipo de productos, tanto a nivel de producción como a nivel comunicativo. A través del análisis de contenido de los mensajes de sus spots y de un grupo de discusión, se ha procedido a realizar un estudio comparativo de los mismos entre la década del ochenta y los spots de los últimos años para ver su evolución.

Palabras clave: Comunicación persuasiva, publicidad, bollería infantil, televisión, mensaje, código PAOS.

Abstract: In recent years, a great diversity of new products has emerged in the sector of children's pastries, but there are others that have long been in our daily lives. In spite of being more those that have failed than those that are maintained, it is crucial to analyze how its advertising has evolved over time, knowing how to adapt to the tastes of different generations of consumers. There has clearly been a movement of concern and awareness among the customers and consumers about the ingredients that make up the food, and because of the change in attitude and the proliferation of consumer rights associations, 
there has been an evolution in this type of products, both at the production level and at the communicative level. Through the content analysis of the messages from their spots and a discussion analysis, a comparative study of the same between the years of the eighties and the last years has been made to see their evolution.

Key words: Persuasive Communication, Advertising, Children's Pastry, Television, Message, PAOS Code.

\section{Introducción}

La publicidad es una forma de comunicación persuasiva para ayudar a vender productos, diferenciando unos de otros, pero que sin embargo puede influenciar de manera muy profunda en la sociedad y en las motivaciones de los individuos. En un principio se intentaban cubrir necesidades más o menos básicas, pero más adelante se han ido creando necesidades nuevas.

Dicho esto, la publicidad intenta satisfacer necesidades de las personas mediante la compra de productos. Esto produce un estado de alivio temporal en los sujetos, pero que tiene una duración muy breve, llegando a ser cada vez más corta. Las empresas aprovechan esto para bombardear a los consumidores con productos nuevos o parecidos a los ya existentes, intentando que estos compren, independientemente de si la necesidad es real o no. Uno de los peligros de la publicidad es que no diferencia realidad de ficción y eso puede llegar a crear confusión en los espectadores, quienes buscan cada vez más la satisfacción rápida de deseos inmediatos.

Es por ello que resulta necesario la existencia de leyes y códigos deontológicos que permitan controlar la publicidad en determinados sectores (como en la bollería infantil) para evitar actos ilegales o inmorales, defendiendo así a los ciudadanos y a los grupos más frágiles de la población. Dentro de los ciudadanos hay personas más sensibles a la publicidad como los niños y ancianos. Los niños son fácilmente influenciables ya que todavía no tienen desarrollada su personalidad ni su manera de ser. Los ancianos, por su parte, no han estado tan expuestos a la publicidad como las personas de mediana edad, por lo que el bombardeo publicitario les afecta muy directamente.

Resulta por tanto interesante realizar un estudio sobre la comparativa de los primeros spots de bollería infantil de los ochentas y comprobar su evolución y cambio a las normas y sociedad del siglo XXI.

\section{Objetivos de investigación}

A continuación se destacan los siguientes objetivos principales:

- Conocer la evolución de los mensajes de los spots del sector de la bollería infantil en España desde los ochentas, época dorada de la publicidad en televisión. 
- Determinar y valorar si han existido cambios importantes en la visualización del target y de los valores transmitidos en la comunicación persuasiva de los spots desde los ochentas hasta el año 2016 principalmente.

\section{Metodología de estudio}

Para la elaboración de la parte empírica se ha llevado a cabo un análisis de contenido y un grupo de discusión. Ambas técnicas escogidas son correctas y adecuadas, ya que se han podido analizar spots de las mismas marcas desde los mismos criterios y puntos de vista (duración, claims, mensaje, valores difundidos). El grupo de discusión ha aportado valiosa información para un análisis final desde la perspectiva subjetiva del consumidor y para comprender la situación de primera mano. De esta forma, se validaba la investigación cuantitativa con la cualitativa.

La finalidad era analizar y comparar los spots de las mismas marcas y productos en los ochentas con los de la actualidad (2014-2016). Así, se podría determinar si se han hecho cambios en la comunicación en televisión y si a través de sus claims y valores difundidos se han adaptado a los códigos éticos, normas de alimentación saludable y adaptación a los cambios en la nueva sociedad.

Las marcas que se han sometido al análisis de contenido han sido las que se muestran abajo en la figura 1. Se muestra su contenido energético y además, son las más populares y consumidas entre la población infantil en el año 2016.

Figura 1: Marcas de bollería sometidas al análisis de contenido y comparativa entre diferentes décadas (1980-2016).

\begin{tabular}{|c|c|c|c|c|c|c|}
\hline MARCAS & Tigretón & Bony & Phoskitos & Donuts & Donettes & Bollycao \\
\hline $\begin{array}{c}\text { Valores } \\
\text { energéticos }\end{array}$ & $1568 \mathrm{~kJ}$ & $1537 \mathrm{~kJ}$ & $1757 \mathrm{~kJ}$ & $1723 \mathrm{~kJ}$ & $1988 \mathrm{~kJ}$ & $1544 \mathrm{~kJ}$ \\
\cline { 2 - 7 } & $373 \mathrm{kcal}$ & $365 \mathrm{kcal}$ & $419 \mathrm{kcal}$ & $412 \mathrm{kcal}$ & $476 \mathrm{kcal}$ & $368 \mathrm{kcal}$ \\
\hline Grasas & 12 & 11 & 18 & 23 & 28 & 14 \\
\hline $\begin{array}{c}\text { Ácidos grasos } \\
\text { saturados }\end{array}$ & 9.2 & 6.9 & 13 & 12 & 17 & 4.7 \\
\hline $\begin{array}{c}\text { Hidratos de } \\
\text { carbono }\end{array}$ & 62 & 62 & 58 & 46 & 50 & 52 \\
\hline Azúcares & 42 & 38 & 39 & 20 & 27 & 24 \\
\hline Proteínas & 3.4 & 3.6 & 5 & 5.3 & 6 & 7.2 \\
\hline Sal & 0.4 & 0.8 & 0.23 & 0.5 & 0.9 & 0.5 \\
\hline
\end{tabular}

Fuente: Elaboración propia. 
Por otro lado, el grupo de discusión de 6 personas ayudó a mostrar (desde un punto de vista más subjetivo) la visión de los spots de las marcas de los ochentas y cómo habían cambiado en la actualidad para ofrecer una imagen y un mensaje más acorde con las necesidades y las exigencias del mercado actual. A pesar de ser un grupo reducido, la información apoyó los resultados obtenidos posteriormente de la plantilla de análisis, en la que se analizaron valores difundidos, target visualizado, claim, duración del spot y estrategia. De esta manera, se pudieron obtener resultados más convincentes y completos de la investigación global.

\section{Hipótesis de trabajo}

En la investigación se ha partido de dos hipótesis interrelacionadas con el mensaje persuasivo de la comparativa de los spots en televisión, siendo las siguientes:

- Los spots narrativos de las marcas analizadas muestran un target diferente en los últimos años, frente a los emitidos en los ochentas.

- La duración de los mismos (spots) es más breve en los últimos años 2014-2016, utilizando un claim más directo y corto.

\section{Sector de la alimentación en España}

Han pasado casi 50 años desde que se inició un movimiento dedicado a la transformación de la nutrición en una especialidad dentro del ámbito de la medicina. Cobra una especial importancia la pediatría, es decir, el tratamiento de los niños hasta su adolescencia y etapa de especial importancia y crecimiento en los seres humanos.

Resulta crucial analizar la situación de la publicidad en España en los ochentas. Para ello es necesario remontarse a los setentas. El país vivió durante muchos años en una dictadura restrictiva, por lo que hasta 1985, diez años después de la muerte de Franco, no se abrieron las fronteras en su totalidad. Eso hace que la publicidad dentro de nuestro territorio fuese dirigida a un público no muy amplio, aunque la mayor parte de los ciudadanos veían casi de manera exclusiva dos canales de televisión. Gracias a esta apertura de fronteras, se inició una liberación de los valores y las costumbres socialmente habituales hasta entonces (Nestlé, 2005).

Cobran una especial importancia los educadores, quienes enseñan a los jóvenes a alimentarse de manera razonable e intentando reducir los problemas de salud. Sin embargo, en los últimos tiempos se han expandido las denominadas "pandemias del siglo XXI", conocidas como la obesidad y otras que derivan de esta: la diabetes, la hipertensión, los problemas metabólicos en general, el aumento de las alergias, la enfermedad celíaca, así como el cáncer. Además de los padecimientos físicos, es necesario prestar también atención a los problemas psicológicos, como la bulimia y la anorexia (OMS, 2016). 


\subsection{Sector de la bollería hasta la actualidad}

Andrades (2015) establece que algunos de los pastelitos más populares y consumidos durante la Educación General Básica (EGB) eran el Bollycao y Phoskitos. La bollería apareció en el mercado español a mediados de los sesentas, pero la fama y ventas de estos productos despegaron en los ochentas gracias a los anuncios de televisión y a las promociones de cromos, pegatinas y calcomanías que se incluían en el interior de estos productos. Los primeros en aparecer fueron de la empresa Panrico, con la creación de la marca Donuts en 1964. Más tarde surgió Bimbo en 1966 con los productos Tigretón, Bony y Pantera Rosa. Después, apareció la gran empresa internacional Adam Foods con la creación de Phoskitos y en 1975 Panrico comenzó a producir Bollycao. El último en incorporarse a esta lista fue Donettes, creado en 1976 (Andrades, 2015).

Las empresas que forman parte de ASEMAC (Asociación Española de la Industria de Panadería, Bollería y Pastelería) han afirmado que en 2016 han aumentado su producción un 5\% respecto al año 2015. La facturación se encuentra en los 1183 millones de euros, aunque la mayor parte de estas cifras viene referida a la producción de pan (Núñez, 2015).

Concretamente, en producción de bollería y pastelería, se ha pasado de 134 mil a 144 mil kilos (aumentando un 7.5\%) superando al incremento respecto al pan. En cuanto a la facturación de bollería y pastelería, se ha pasado de 364 mil a 395 mil euros, aumentando su facturación en un $8.5 \%$. Estas cifras son muy positivas para el sector: pese a las campañas de reducción de consumo de estos productos y la crisis económica, siguen produciéndose y vendiéndose en abundancia. Gran peso en la producción de bollería la tienen Bimbo, Adam Foods y Panrico (Pérez-Reverte, 2016).

\subsection{Hábitos de consumo de la bollería en España}

Statista (2017) refleja la cuota de mercado en la comercialización de bollería, pastelería, galletas y cereales por canales de venta en hogares de España en 2014. Como se observa en la figura 2, el número de tiendas tradicionales ha descendido a favor de los supermercados e hipermercados ${ }^{1}$ donde se prefiere la compra de las marcas más conocidas de bollería entre la población infantil. La tendencia (como puede verse) trata de un incremento de las grandes superficies que ofrecen una amplia variedad de productos.

1 La diferencia entre hipermercado y supermercado radica en su superficie de venta, ya que en el primer caso el espacio supera los $2500 \mathrm{~m}^{2} \mathrm{y}$, en el segundo caso, el espacio está comprendido entre los 400 y los $2500 \mathrm{~m}^{2}$ (Adam Foods, 2015). 
Figura 2: Evolución del número de establecimientos en España (1985-2014).

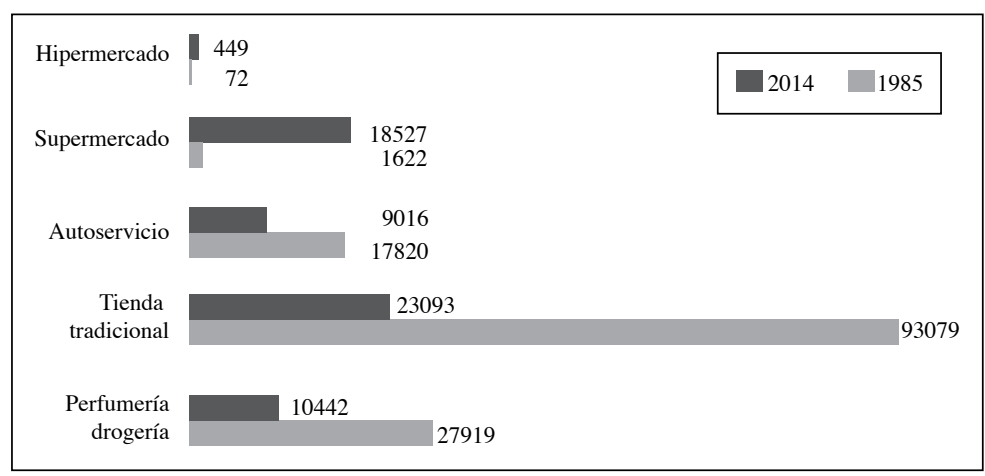

Fuente: Statista (2017).

\subsection{Dónde y cómo se consume bollería}

Según Martín Cerdeño (2015), existen una serie de factores clave que interfieren en el consumo de la bollería, pastelería, galletas y cereales. El primer factor clave es la condición económica, ya que en los hogares de clase alta y media alta, tienen un mayor consumo en este tipo de productos (un 13\% por encima de la media). Los hogares de clase baja, sin embargo, tienen una demanda de un $16.4 \%$ inferior a la media. Podría entenderse que la bollería no es un producto de primera necesidad.

Otro factor muy importante es la presencia de niños en el hogar. El consumo de bollería y pastelería a granel es un 15\% superior en los hogares donde no hay niños, mientras que el consumo de galletas dulces y cereales es superior en los hogares donde sí los hay.

El nivel económico y social también es una pieza clave en este aspecto, ya que si la persona encargada de hacer la compra no trabaja, el consumo de bollería, pastelería, galletas y cereales es superior en un $2,3 \%$. Esto puede ir relacionado con el aprendizaje para comer saludablemente y la aplicación de una rutina en el día a día.

También resulta importante la edad, ya que donde compra una persona de más de 65 años, el consumo de bollería, pastelería, galletas y cereales es un $2,7 \%$ más elevado. Resulta interesante saber que donde la demanda es más reducida es en los hogares cuyo comprador principal es alguien menor de 35 años, teniendo un consumo inferior al 14,7\% respecto a la media (Ocu, 2016).

\subsection{Códigos deontológicos}

Existen numerosos códigos deontológicos respecto a cómo enfocar la publicidad de productos destinados a la población de menor de edad en España. Los nuevos hábitos de com- 
portamiento de la sociedad moderna han supuesto un incremento nunca visto en cuanto al sedentarismo y el déficit de gasto energético de la población. Todo ello ha generado un aumento de la obesidad y sobrepeso, del cual no se puede hacer completamente responsable a la industria de la alimentación, sino a las instituciones, los tutores y los propios afectados por ello. Esto ha generado la necesidad de llegar a acuerdos entre todos para fomentar un estilo de vida más saludable.

Según la Organización Mundial de la Salud, 1 de cada 3 niños menor de 11 años padece sobrepeso o está dentro de los márgenes de obesidad. La prevalencia de la obesidad se ha duplicado entre el año 1980 y 2008, llegando a incrementarse los índices de obesidad desde edades muy tempranas, tanto en niñas como en niños en general. Según las últimas estimaciones, en los países europeos el sobrepeso afecta hasta a un $70 \%$ de los adultos y la obesidad hasta a un $30 \%$ de estos. Estas cifras también son aplicables a los niños, ya que viven influenciados por sus padres, así como por los hábitos que estos poseen (ápud Ministerio de Sanidad del Gobierno de España, 2015).

Otra estimación reciente declara que un $60 \%$ de los niños que sufrieron sobrepeso antes de la pubertad, la seguirán padeciendo en su edad adulta (Ministerio de Sanidad del Gobierno de España, 2015). Esto acarrea diversas enfermedades como desórdenes alimenticios, baja autoestima, diabetes del tipo 2 e incluso problemas cardiovasculares. La manera de prevenir estos problemas (además de adquirir hábitos saludables y realizar ejercicio con cierta regularidad) radica en los acuerdos entre el gobierno, las comunidades autónomas, los medios de comunicación ${ }^{2}$ y el sector privado. De esta manera, la concienciación social será más elevada.

\subsubsection{Código PAOS}

Un ejemplo de esta protección a la sociedad frente a la publicidad de productos alimenticios es el código de corregulación de la publicidad de alimentos y bebidas dirigidas a menores, prevención de la obesidad y salud, conocido también como el código PAOS (Ministerio de Sanidad del Gobierno de España, 2012). El objetivo de este código es, junto con otras instituciones comunitarias además de la OMS, disminuir la prevalencia de la obesidad y el sobrepeso, además de otras repercusiones tanto sociales como de salud pública. Según la AECOSAN (2016), el 46\% de la población infantil en nuestro país sufre obesidad o sobrepeso, por lo que las consecuencias del sedentarismo y los malos hábitos alimenticios son palpables.

Este código se generó en gran parte gracias a la estrategia NAOS o Estrategia para la Nutrición, Actividad física y Prevención de la Obesidad, lanzada en 2005 por el Ministerio

2 En España, en los últimos años, se pueden observar mensajes en los anuncios de bollería infantil como: "La mejor receta: alimentación equilibrada y ejercicio físico", gracias a un acuerdo llevado a cabo entre el Ministerio de Sanidad y las empresas de Restauración Moderna. 
español de Sanidad y Consumo y la Federación de Industrias de Alimentación y Bebidas (FIAB), cuyo objetivo era disminuir la prevalencia de obesidad y sobrepeso y sus consecuencias, tanto en el ámbito de la salud pública como en sus repercusiones sociales. En resumen, el código PAOS son una serie de normas o reglas que sirven de guía a la hora de realizar mensajes publicitarios cuando van destinados a niños menores de 12 años y cuando sean retransmitidos en televisión y, de menores de 15 años los publicados en la Internet.

\subsubsection{Autocontrol: Asociación para la autorregulación de la comunicación comercial}

Este organismo tiene como su principal misión: el deseo de que anunciantes, agencias y medios en España trabajen juntos por una publicidad veraz, legal, honesta y leal. De esta forma, se configura como "un órgano extrajudicial de resolución de reclamaciones en materia publicitaria compuesto por expertos de prestigio" (Autocontrol, 2012) en diferentes campos relacionados con la publicidad, como el derecho, la comunicación comercial y el marketing. Cualquier interesado puede reclamar, desde asociaciones de consumidores, empresas o asociaciones empresariales, consumidores y organismos de administraciones públicas. El objeto de reclamación puede ser cualquier anuncio concreto difundido en cualquier medio dentro de las fronteras de España durante el año anterior que se entienda que infringe las normas éticas de los códigos de conducta y, por lo tanto, las normas legales (Autocontrol, 2012).

Figura 3: Actividad preventiva de los anuncios.

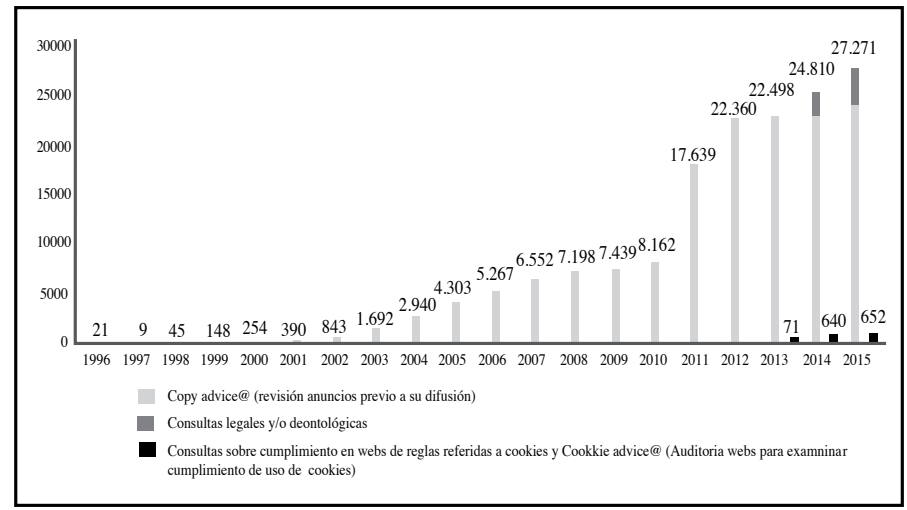

Fuente: Autocontrol (2016).

\section{Resultados}

\subsection{Análisis de contenido y comparativa de spots (1980-2016)}

A continuación se muestran los resultados más destacables de las dos técnicas empleadas. En la figura 4 se puede ver que la duración de todos los spots es mucho menor que en los ochentas, siendo más sintéticos y visuales, con un claim más corto y directo. 
Figura 4: Comparativa de la duración de spots (1980-2016).

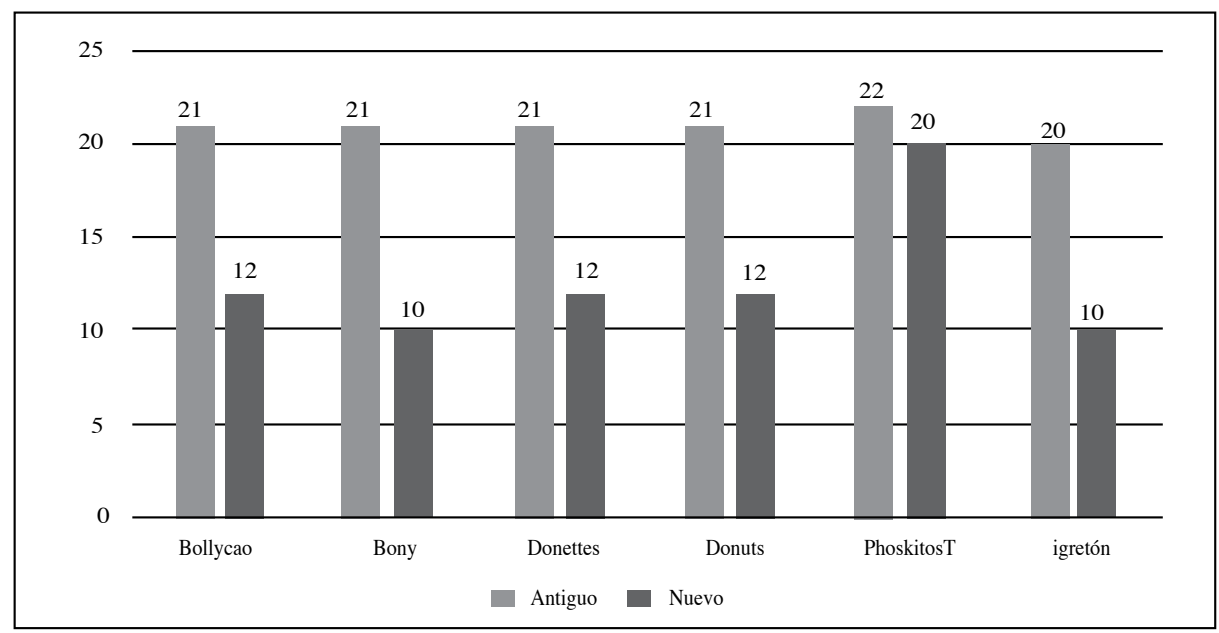

Fuente: Elaboración propia.

Como se observa en el spot (2016) de las marcas Tigretón y Pantera Rosa (figura 5), se alude a la nostalgia de los que consumían dicha marca en los ochentas. Muchas de las marcas que nacieron en esa década vuelven a recurrir en ese tipo de mensajes de los primeros consumidores que hoy ya se acercan a la cuarentena de edad. De esta forma, se consigue una capacidad de fidelización más elevada.

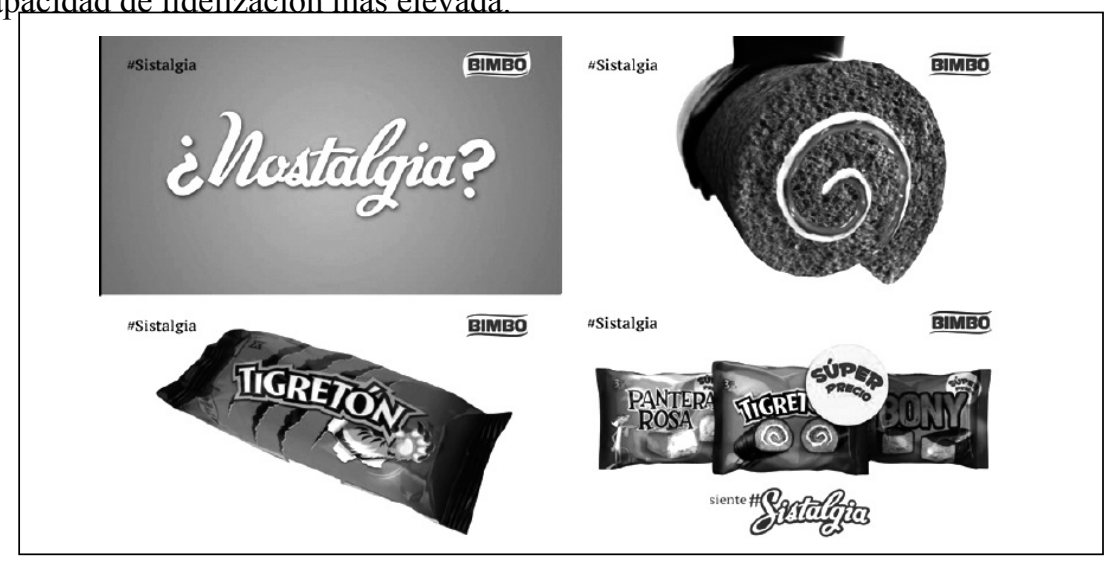

Fuente: YouTube (2016).

Por otro lado, en la siguiente spot (figura 6) se juega únicamente con la animación de dibujos dirigida a un público infantil, viéndose la marca representada a través de los personajes protagonistas: Bony y Tigretón. 
Figura 6: Spot Bony y Tigretón (1993).

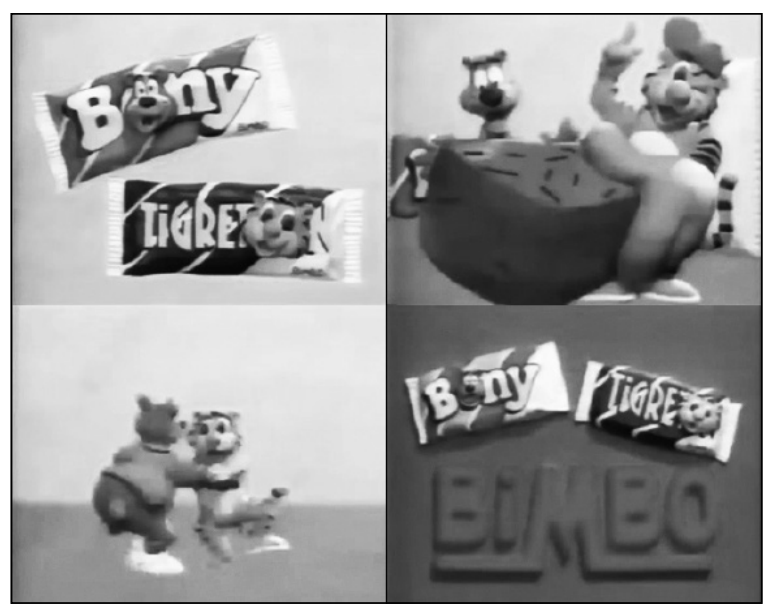

Fuente: YouTube (2013a).

En el siguiente spot la marca Donuts (figura 7) muestra de forma sencilla al propio consumidor (público infantil), en un momento del consumo del producto que bien podría ser el desayuno.

Figura 7: Spot Donuts (1981).

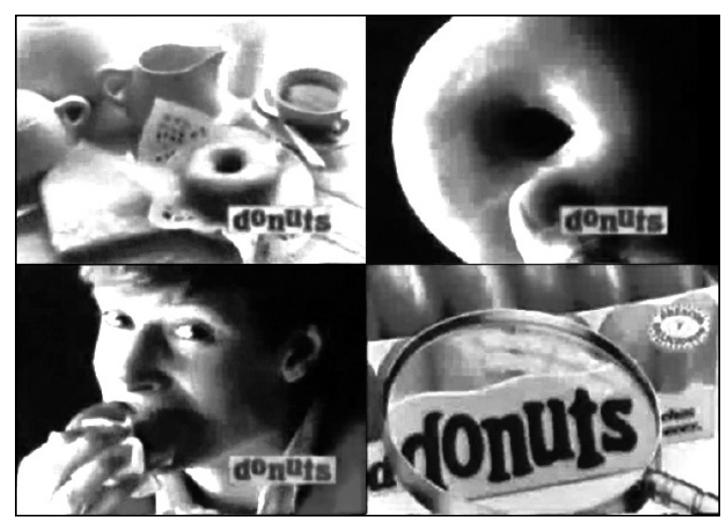

Fuente: YouTube (2014a).

En otro spot de la misma marca (figura 8), se observa que la edad del target se ha elevado. Este factor y estrategia que están llevando estas marcas de bollería es muy común en los últimos años; ya que para conseguir y mantener a los primeros consumidores de las marcas en sus inicios y fortalecer ventas, deben mostrar a un público más adulto que en los ochentas consumieron estas marcas de bollería y que en la actualidad podrían seguir haciéndolo. Es por ello que este spot se hace un guiño a la nostalgia para un público más maduro y a la vez diverso. 
Figura 8: Spot Donuts (2016).

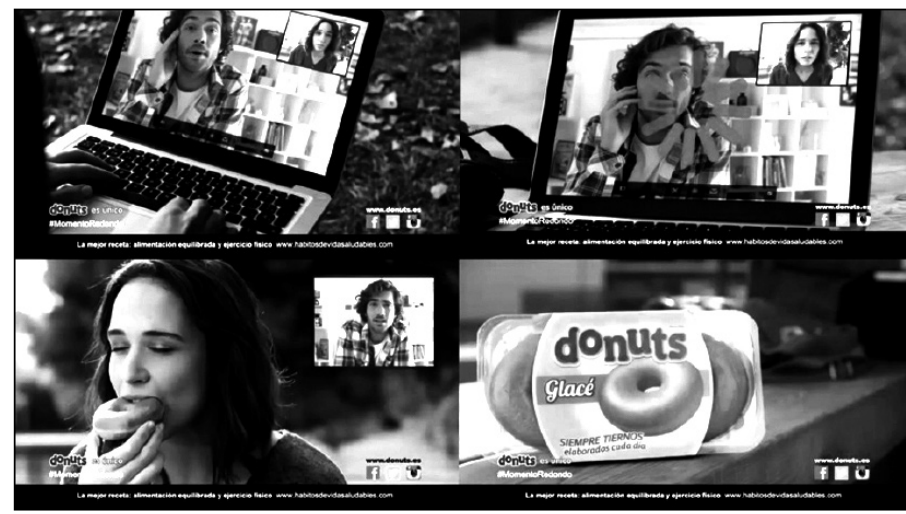

Fuente: YouTube (2017).

En definitiva, las marcas de bollería infantil (que se remontan a los ochentas) han adaptado sus mensajes, imágenes y claims a la actualidad del mercado y de sus consumidores del siglo XXI, fundamentando y reforzando sus estrategias, no sólo en un tipo de consumidor infantil, sino también enfatizando los componentes e ingredientes de los productos como valor saludable para su consumo.

Figura 9: Spot Donettes (1987).

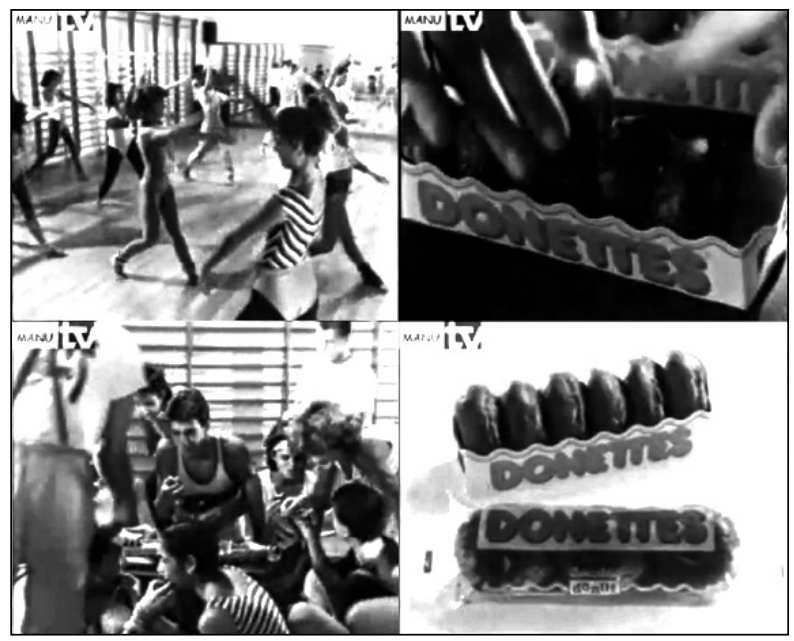

Fuente: YouTube (2013b).

Además de esto, dichas marcas analizadas como Donettes (figuras 9 y 10) siguen ponderando valores como la amistad y el cariño entre los amigos y la familia: valores aún muy arraigados y considerados positivamente en nuestra sociedad actual. 
Figura 10: Spot Donettes (2014).

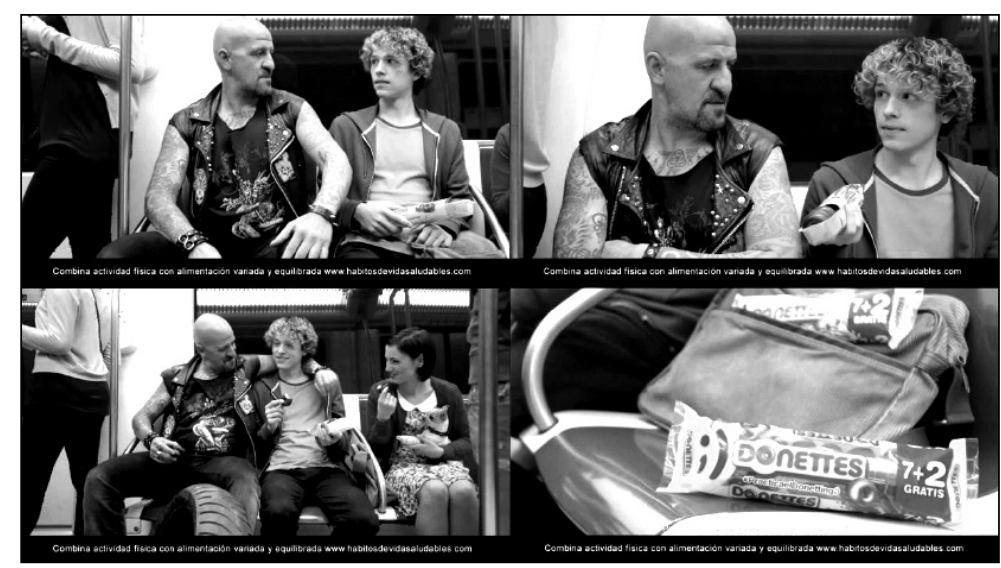

Fuente: YouTube (2014b).

\subsection{Resultados grupo de discusión}

El grupo de discusión estuvo conformado por seis personas, reunidas en una misma sala de una universidad privada de Valladolid (España). Tres de ellas fueron de edad avanzada (sobre los 55-60 años) y que en la actualidad son abuelos y fueron padres en los ochentas, por lo que han sido posibles compradores de los productos por un largo período de tiempo. Las otras tres personas rondaban los 38-40 años, por lo que de pequeños es muy probable que consumiesen los productos analizados y en la actualidad se los ofrezcan a sus hijos o incluso los consumen ellos mismos de forma ocasional.

Se les reunió en una misma sala, donde se les proyectó una primera tanda de anuncios de los ochentas sobre las marcas comentadas con anterioridad (figura 1) junto con algunas preguntas sobre opinión de valores transmitidos y mensaje del spot. Más tarde se les proyectó una segunda tanda de las mismas marcas, pero de años posteriores (2014-2016) con las mismas preguntas.

Entre ambas generaciones de encuestados se pudieron observar bastantes opiniones coincidentes, como el desacuerdo de la técnica de Phoskitos de no vender el producto, sino de vender un juguete o regalo. Todos coincidieron en que era una técnica peligrosa que inducía a engañar a los niños para comprar un juguete y no un producto de alimentación, llegando a sugerir la prohibición de esta técnica.

Asimismo, existieron discrepancias sobre a quiénes iban dirigidos los anuncios, ya que algunos se sentían identificados y otros los veían claramente hechos para niños. En su mayoría, opinaron que la marca Donuts, en su anuncio de 1981, seguía una línea dirigida más a adultos, ya que son quienes más aparecen en el anuncio, pero también por el tipo y estilo de música. 
En general, gustaron bastante los anuncios de Donettes porque iban dirigidos a un público más juvenil y ensalzaba valores como la amistad, aunque a un sujeto no le gustó que en el anuncio de 2014 se compartiese el producto "a modo de chantaje" para evitar una especie de pelea. Tras expresar su opinión, el resto de personas le dieron la razón y pasaron a valorar como mejor el anuncio de Donettes de 1987 frente al del año 2014, porque no aludía indirectamente a la violencia.

Los spots que pasaron de forma más desapercibida fueron los de Bony y Tigretón, aunque sí generaron una sensación de nostalgia en la generación que contaban con 38-40 años. La generación mayor no llegó a entender el concepto de "nostalgia" y al no haber consumido esos productos de niños, tampoco les impactó demasiado. Algo similar ocurrió con la marca Bollycao, cuyo cambio de imagen (al incorporar información sobre su composición en hierro) causó una buena impresión en todos los sujetos, salvo en uno que opinó que quienes consumían dichos productos era por su sabor, independientemente de su composición.

\section{Conclusiones}

Cabe decir que tan solo la mitad de los anuncios analizados (6 marcas) hacen referencia a las características del producto. Si se quiere ampliar información es necesario ir al punto de venta y analizar sus etiquetas o buscarlos en la Internet. La información nutricional no aparece en ninguno de los spots y únicamente el spot más nuevo (del año 2016) de Bollycao, hace referencia a un componente de sus ingredientes: el hierro.

La mitad de los anuncios hablan de las características que precisamente los hacen diferentes de los demás, como por ejemplo que son "tiernos" (en el caso de Donuts) o algo clásico y sano (Bollycao). Sin embargo, la otra mitad de los productos dan por sentado que la gente que los compra ya saben cómo son o que simplemente no les importa su composición. Este es el caso de las marcas Bony, Tigretón, Donettes y Phoskitos.

El storytelling de los spots es muy simple, ya que siempre se presenta al producto como algo fácil, cómodo, divertido o de moda, que soluciona siempre de manera rápida y sencilla el momento de hambre a media tarde (se suelen presentar para el momento de la merienda). Bollycao es representado (en ambas épocas) como la solución a una merienda simple, tradicional y no tan "insana" como se puede llegar a pensar. Bony y Tigretón hacen referencia a la primera época a la infancia, tema que se repite en el anuncio moderno como algo bueno. En otras palabras, es un producto que te hace feliz porque te ayuda a recordar una infancia en la que fuiste feliz. Donettes siempre ensalza la amistad entre adolescentes, en las que el producto se puede compartir. Donuts ha pasado de dejar claro a la audiencia que es un producto único e inimitable, a ser un producto con tradición que nos acompaña a lo largo de muchos momentos de nuestra vida. Phoskitos habla muy poco o prácticamente nada de su producto y pasa a describir directamente el regalo con el que viene y lo divertido que es.

Tan solo un anuncio usa dibujos animados a lo largo de toda su duración y únicamente uno añade un dibujo durante unos segundos dando información nutricional. Podría inter- 
pretarse como que el hecho de no incluir dibujos, pese a poder ser un producto pensado para los niños, aporta seriedad en torno a la empresa y da mayor credibilidad. Si bien es cierto que, en la actualidad, los niños cercanos a los 8 años empiezan a ver programas o series representados por adultos (y los dibujos animados han pasado a ir dirigidos a niños muy pequeños, menores de 7 años) los dibujos animados han pasado a vincularse con algo extremadamente infantil.

Los personajes representados son muy variados, observándose adultos, niños, adolescentes y (en dos casos concretos) únicamente el producto. Esto crea la sensación de que la bollería no es solo un producto para niños, sino que cualquier adulto puede disfrutar de ello. Además, aporta seriedad y credibilidad entorno al producto, siendo representado como algo que tienen en común todas las generaciones de la sociedad, independientemente de su formación académica o sus conocimientos tecnológicos.

Los valores a los que más se recurren son la juventud, la modernidad y el éxito, aunque también se encuentran otros como la tradición y el entretenimiento. La sensación que se quiere transmitir es que en el presente se puede tomar un producto del futuro, que está bueno y además, si mucha gente ha crecido con ello a lo largo de su vida, será porque es saludable y bueno.

La preocupación por el consumo de estos productos se ve reflejado en la estandarización de un rótulo a modo de impresión que aconseja hacer ejercicio físico para tener una vida sana, así como el hecho de comentar algunos componentes beneficiosos, como es en el caso de la marca Bollycao, con el claim establecido. "Meriendas de cacao y hierro". Dicho rótulo se incorpora debido al Plan Havisa: un convenio de colaboración para el desarrollo de un plan de fomento de hábitos de vida saludables, firmado por la Aecosan y la fundación Alimentum. La campaña está orientada a la televisión, dando visibilidad a mensajes dirigidos a la promoción de hábitos de vida saludables y que va en consonancia con la estrategia Naos (Puro Marketing, 2014).

También se puede observar que Bollycao ha cambiado su imagen, pasando de dirigirse a los niños (diciendo que "está rico"), a dirigirse a las madres, informando que tiene un alto contenido en hierro. Sin embargo, los valores de juventud de Donettes y de entretenimiento de Phoskitos se encuentran presentes en todo momento en ambas generaciones de anuncios. Los niños han quedado (en general) en un segundo plano y ahora se intenta captar la atención de los adultos, independientemente de si son quienes consumen el producto o no. Esto puede deberse a que en la sociedad española hay un mayor número de adultos que de niños y que las tasas de natalidad en los últimos años han descendido notablemente, olvidando el "baby-boom" de los ochentas.

Por otro lado, la creación de nuevas líneas de productos que añaden el término "light" da a entender que el sector de la bollería no va a extinguirse a largo plazo y que está sufriendo una renovación en el cambio de composición de sus productos. Todavía continúa la percepción de que son productos insanos y poco saludables. Son muchos los estudios que analizan el efecto a largo plazo de estos productos y es innegable que su uso abusivo resulta 
devastador. Sin embargo, también lo es comer únicamente hamburguesas o únicamente verduras y frutas. No obstante, lo que sí es cierto es que la comunicación en televisión de estas marcas analizadas ha ido cambiando en función de las exigencias del mercado, hábitos de consumo y normas y códigos éticos que favorecen un estilo de vida más saludable y equilibrado (Baños y Rodríguez, 2012).

Por todo lo que se ha analizado en las marcas comparadas, queda de manifiesto que la estructura narrativa de los spots de los últimos años es más directa, corta en duración de minutos y muestra un target más amplio, no únicamente a un público infantil sino más adulto que también consume en algún momento este tipo de productos y marcas.

Es por ello que la publicidad tiene como papel en la sociedad cumplir la misión de transmitir valores saludables del cuidado de nuestra alimentación a través de la comunicación persuasiva de los mensajes de los spots de este sector en cuestión.

\section{Fuentes consultadas}

AECOSAN, Agencia española de Seguridad Alimentaria (2016). "Consumo en la alimentación de los españoles". Extraída el 2/II/2017 desde

http://www.aecosan.msssi.gob.es/AECOSAN/web/enlaces/aecosan_enlaces.htm\#org_autonomicos_consumo

Adam Foods (2015). “¿Quiénes somos?” Extraída el 14/VI/2017 desde http://www.adamfoods.com/es/nuestra-empresa/quienes-somos

Andrades, A. (2015). "Los pastelitos de la EGB: Bollycao, Phoskitos y Pantera Rosa". Extraída el 15/VI/2017 desde

http://cincodias.com/cincodias/2015/06/05/sentidos/1433532089_452098.html

\section{Autocontrol, Asociación para la Autorregulación de la Comunicación Comercial.} _(2016). “Autocontrol de la publicidad. Balance actividad 2015”. Extraída el 6/XI/2017 desde http://www.autocontrol.es/pdfs/balance_15_AUTOCONTROL.pdf

_(2012). “Autocontrol de la publicidad. Trabajamos por una publicidad responsable". Extraída el 18/X/2017 desde http://www.autocontrol.es/pdfs/balance\%2011\%20autocontrol. pdf

Baños, M. y Rodríguez, T. (2012). "Imagen de marca y product placement". Extraída el 20/VI/2017 desde

https://books.google.es/books?id=S9cIDQAAQBAJ\&printsec=frontcover\&hl=es\&source $=\mathrm{gbs} \_g e \_s u m m a r y \_r \& c a d=0 \# \mathrm{v}=$ onepage $\& \mathrm{q} \& \mathrm{f}=$ false

Martín Cerdeño, V. (2015). “Consumo de dulces en España”. Extraída el 9/VI/2017 desde http://www.mercasa.es/files/multimedios/1425470661_Consumo_de_dulces_en_ Es pana.pdf 


\section{Ministerio de Sanidad, Servicios Sociales e Igualdad del Gobierno de España.}

_(2015). "Sanidad y las empresas de la Restauración Moderna acuerdan colaborar en un plan de promoción de estilos de vida saludables en la población española”. Extraída el 16/ VI/2017 desde http://www.msssi.gob.es/gabinete/notasPrensa.do?id=3627

_(2012). "Código de corregulación de la publicidad de alimentos y bebidas dirigida a menores, prevención de la obesidad y salud (Código PAOS)". Extraída el 17/X/2017 desde http://www.aecosan.msssi.gob.es/AECOSAN/docs/documentos/nutricion/Nuevo_Codigo_PAOS_2012_espanol.pdf

Nestlé (2005). Una historia de la publicidad española. Reflejos de más de un siglo de Nestlé. Barcelona: Taller Editorial Mateu.

OCU (2016). “Datos sobre consumo en el sector de la bollería industrial”. Extraída el 25/ III/2017 desde https://www.ocu.org/alimentacion

OMS, Organización Mundial de la Salud (2016). "Trastornos alimenticios en los jóvenes". Extraída el 20/II/2017 desde http://www.who.int/topics/es/

Pérez-Reverte, A. (2016). "La merienda del niño". Extraída el 17/X/2017 desde https:// www.zendalibros.com/la-merienda-del-nino/

Puro Marketing (2014). "La historia de Donuts, una marca y el agujero más famoso de la historia”. Extraída el 6/VI/2017 desde http://www.puromarketing.com/3/18342/historiadonuts-marca-agujero-famoso- historia.html

Statista (2017). "Porcentaje de ventas de bollería, pastelería, galletas y cereales por formatos para hogares en los distintos canales de distribución en España en 2015”. Extraída el 17/X/2017 desde

http:/es.statista.com/estadisticas/501706/cuota-de-mercado-en-la-comercializacion-debolleria-y-pasteleria-en-espana/

\section{YouTube}

_(2017, abril 5). “Donuts, tu momento redondo”. Extraída el 2/XI/2017 desde https://www. youtube.com/watch?v=6wUhEBPfWI8

_(2016, enero 18). "Spot Tigretón - Sistalgia". Extraída el 2/XI/2017 desde https://www. youtube.com/watch?v=bHdWGqca1CA

_(2014a, junio 4). “Dunkin' Donuts 1981 Commercial”. Extraída el 2/XI/2017 desde https://www.youtube.com/watch?v=-ffUjAuoIlA

_(2014b, abril 11). “Anuncio Donettes”. Extraída el 2/XI/2017 desde https://www.youtube. com/watch? $\mathrm{v}=\mathrm{x} 0 \mathrm{iAFQVN} 48 \mathrm{U}$

_(2013a, diciembre 4). "Spot Bony y Tigretón 'Somos Bony y Tigretón'”. Extraída el 2/ XI/2017 desde https://www.youtube.com/watch?v=BIjaxHNsDMw _(2013b, marzo 13). “Anuncio Donettes. Conductor de primera”. Extraída el 2/XI/2017 desde https://www.youtube.com/watch?v=4LQYbendhVA 